\title{
CONVOLUTION EQUATIONS IN SPACES OF DISTRIBUTIONS SUPPORTED BY CONES
}

\author{
ALEX MERIL AND DANIELE C. STRUPPA
}

\begin{abstract}
We describe some examples of surjective convolutors on $D^{\prime}(\Gamma)$, for $\Gamma$ a closed convex cone in $\mathbf{R}^{n}$. We also give necessary and sufficient conditions on $S_{1}, \ldots, S_{m}$ in $S^{\prime}(\Gamma)$ to be generators of the whole convolution algebra $S^{\prime}(\Gamma)$.
\end{abstract}

0. Introduction. Let $\Gamma \subset \mathbf{R}^{n}$ be a closed convex cone, whose vertex is the origin, and which does not contain any straight line. Denote by $D^{\prime}(\Gamma)$ the space of distributions supported in $\Gamma$; it is well known that $D^{\prime}(\Gamma)$ is a convolution algebra, so if $S \in \mathcal{E}^{\prime}\left(\mathbf{R}^{n}\right)$ is a distribution with compact support contained in $\Gamma$, then $S$ * $D^{\prime}(\Gamma) \subseteq D^{\prime}(\Gamma)$ (see, e.g., [6, p. 33]). By now, a complete characterization (in terms of the decrease of $\hat{S}$, the Fourier transform of $S$ ) of those $S$ for which $S * D^{\prime}(\Gamma)=$ $D^{\prime}(\Gamma)$ is well known (see, e.g., [4, pages 356 and following]). On the other hand, in a recent paper, $[8]$ a characterization of a completely different nature is given, at least for $n=1$ (and hence $\Gamma=[0,+\infty)$ ); this characterization, unfortunately, is of difficult application, as it requires the test of the singular support of $S * u$ for an extremely large class of distributions $u$ and, as a consequence, no nontrivial examples are given in [8].

The purpose of this paper is twofold: first, in $\S 1$, we show that the condition of Shambayati and Zielezny [8] can be replaced by an equivalent one, in which only compactly supported distributions are used, and which has the advantage of being quite easy to check; in particular we are able to use our condition to provide several examples of distributions $S$ in $\mathcal{E}^{\prime}(\mathbf{R})$ for which $S * D^{\prime}([0,+\infty))=D^{\prime}([0,+\infty))$. One can now use this characterization, together with the well-known results of Hörmander on hyperbolic convolution equations, to deduce nontrivial properties of the Fourier transform of those invertible distributions $S \in \mathcal{E}^{\prime}(\mathbf{R})$ for which $\operatorname{sing} \operatorname{supp}(S)=\{0\}$, and of other classes of examples. In this section we also prove that the original characterization provided in $[8]$ can indeed be extended to the case $n>1$; here, however, as we show with a simple example taken from the theory of constant coefficients partial differential operators, the condition cannot be eased.

In $\S 2$, finally, we deal with the following related problem: let $S^{\prime}(\Gamma)$ denote the space of tempered distributions, with support contained in the cone $\Gamma \subset \mathbf{R}^{n}$, and let $S_{1}, \ldots, S_{m}$ be $m$ distributions in $S^{\prime}(\Gamma)$; we provide a necessary and sufficient condition on $\left(S_{1}, \ldots, S_{m}\right)$ in order to ensure the existence of $m$ distributions $E_{1}, \ldots, E_{m} \in S^{\prime}(\Gamma)$ such that $S_{1} * E_{1}+\cdots+S_{m} * E_{m}=\delta$; for $m=1$ the condition was well known (see [7], but also [9 and 10]), while for $m>1$ we prove it with techniques similar to those used by Hörmander in [3]: our method, indeed, uses $L^{2}$-estimates for solutions of the Cauchy-Riemann equation, together with the

Received by the editors January 30, 1986.

1980 Mathematics Subject Classification (1985 Revision). Primary 46F12; Secondary 44A35. 
characterization of the space $\hat{S}^{\prime}(\Gamma)$, provided in [9], and could be suitably modified to yield the solution for a similar problem in $D_{F}^{\prime}(\Gamma)$, the space of finite-order distributions with support in $\Gamma$.

1. According to Ehrenpreis [2], we say that a compactly supported distribution $S \in \mathcal{E}^{\prime}\left(\mathbf{R}^{n}\right)$ is invertible if, with $\hat{S}$ denoting the Fourier transform of $S$, there are constants $A_{1}, A_{2}, A_{3}>0$ such that for every $\xi \in \mathbf{R}^{n}$ there exists $\eta \in \mathbf{R}^{n}$ satisfying

$$
|\xi-\eta| \leq A_{1} \log (2+|\xi|) \text { and }|\hat{S}(\eta)| \geq\left(A_{2}+|\xi|\right)^{-A_{3}} \text {. }
$$

It is well known that a distribution $S$ in $\mathcal{E}^{\prime}\left(\mathbf{R}^{n}\right)$ is invertible if and only if $S$ * $D^{\prime}\left(\mathbf{R}^{n}\right)=D^{\prime}\left(\mathbf{R}^{n}\right)$.

If $K$ is a compact convex set in $\mathbf{R}^{n}, H_{K}(\xi)=\sup _{x \in K}\langle x, \xi\rangle$ will denote its supporting function; denote by $\sharp$ the set of all supporting functions of compact convex sets (including the empty set, whose supporting function is $-\infty$ ): then, if $S \in \mathcal{E}^{\prime}\left(\mathbf{R}^{n}\right), \mathcal{H}(S)$ denotes the set of all $h \in \mathcal{H}$ such that there is a sequence $\xi_{\nu} \rightarrow \infty$ in $\mathbf{R}^{n}$ with

$$
L_{S}\left(z, \xi_{\nu}\right)=\frac{\log \left|\hat{S}\left(\xi_{\nu}+z \log \left|\xi_{\nu}\right|\right)\right|}{\log \left|\xi_{\nu}\right|}
$$

converging to a plurisubharmonic function with supporting function $h$ [4].

Let us now restrict our attention to the case $n=1$; let $S \in \mathcal{E}^{\prime}(\mathbf{R})$ be a distribution) with compact support, $\operatorname{supp}(S) \subseteq[0,+\infty)$; let $D^{\prime}([0,+\infty))$ be the space of distributions with supports contained in $[0,+\infty)$ and $D_{+}^{\prime}$ the space of distributions with supports contained in $[a,+\infty)$, for some $a$ in $\mathbf{R}$. We have:

Theorem 1. Let $S \in \mathcal{E}^{\prime}(\mathbf{R}), \operatorname{supp}(S) \subseteq[0,+\infty)$. Then $S * D^{\prime}([0,+\infty))=$ $D^{\prime}([0,+\infty))$ if and only if $S$ is invertible and

for all $j$ in $\mathbf{Z}$, for all $a$ in $\mathbf{R}$, there exists $b$ in $\mathbf{R}$ such that for all $h \in \mathcal{H}(S)$ and for all $x \in \mathbf{R}$

$$
h(\xi)+x \cdot \xi \leq H_{[j, a]}(\xi), \text { for all } \xi \text {, implies } x \in[j, b] .
$$

ProOF. In view of Corollary 1 of $[8]$, we only have to show that (1) is equivalent to

$$
\begin{aligned}
& \text { for all } j \in \mathbf{Z} \text { and all } u \in D_{+}^{\prime}, \operatorname{sing} \operatorname{supp}(S * u) \subseteq[j,+\infty) \text { implies } \\
& \text { sing } \operatorname{supp}(u) \subseteq[j,+\infty) .
\end{aligned}
$$

We first notice that condition (2) is equivalent to

$$
\text { for all } j \in \mathbf{Z} \text { and all } u \in \mathcal{E}^{\prime}, \operatorname{sing} \operatorname{supp}(S * u) \subseteq[j,+\infty) \text { implies }
$$$$
\operatorname{sing} \operatorname{supp}(u) \subseteq[j,+\infty) \text {. }
$$

It is clear that (2) implies (3), as $\mathcal{E}^{\prime} \subset D_{+}^{\prime}$. On the orther hand, let (3) hold, $u \in D_{+}^{\prime}$ and $\operatorname{sing} \operatorname{supp}(S * u) \subset[j,+\infty)$ for some $j$. Let $\alpha=\inf \{x: x \in \operatorname{supp}(u)\}:$ if $\alpha \geq j$ we do not need to prove anything, otherwise take $\phi \in C_{0}^{\infty}(\mathbf{R}), \phi \equiv 1$ on $[\alpha, j]$, $\phi \geq 0$, and define

$$
T_{1}=\phi \cdot u \text { and } T_{2}=(1-\phi) \cdot u,
$$

so that $u=T_{1}+T_{2}$. Notice that $\operatorname{supp}\left(T_{2}\right) \subseteq[j,+\infty)$, hence $\operatorname{sing} \operatorname{supp}\left(T_{2}\right) \subseteq$ $[j,+\infty)$. Now

$$
S * T_{1}=S * u-S * T_{2}
$$


notice that both $S * u$ and $S * T_{2}$ are $C^{\infty}$ in $(-\infty, j)$, because $\operatorname{supp}\left(T_{2}\right) \subseteq[j,+\infty)$ and $\operatorname{supp}(S) \subseteq[0,+\infty)$; therefore

$$
\operatorname{sing} \operatorname{supp}\left(S * T_{1}\right) \subseteq[j,+\infty) .
$$

Now $T_{1}$ is of compact support, and hence (3) implies that $\operatorname{sing} \operatorname{supp}\left(T_{1}\right) \subseteq[j,+\infty)$. Finally, as $u=T_{1}+T_{2}$, we get that $\operatorname{sing} \operatorname{supp}(u) \subseteq[j,+\infty)$. The equivalence of (3) and (1) is now an immediate consequence of Theorem 16.3.13 of [4].

Condition (1) or, equivalently, condition (3), enables us to give some classes of examples of surjective convolutors onto $D^{\prime}([0,+\infty))$; none of them were given in [8].

EXAMPLE 1. Let $S$ be a compactly supported invertible distribution with $\operatorname{sing} \operatorname{supp}(S)=\{0\}$; then we can either use Corollary 16.3.15 of [4], or remark that, in this case, the set $\mathcal{H}(S)$ contains only one function, namely the supporting function of the convex hull of the singular support of $S$; in both ways one sees that (1) is satisfied, and therefore $S *$ is a surjection.

EXAMPLE 2. Suppose $\hat{S}$ is an exponential polynomial (this means that $S$ is a distribution with finite support) such that $0 \in \operatorname{sing} \operatorname{supp}(S)$. Then it is well known that $S$ is invertible and Corollary 16.3 .18 of $[\mathbf{4}]$ implies that (1) is satisfied.

EXAMPLE 3 . Let $\mu, \nu \in \mathcal{E}^{\prime}(\mathbf{R}$; suppose that $\mu$ contains an atom, $\operatorname{sing} \operatorname{supp}(\mu) \cap$ $\operatorname{sing} \operatorname{supp}(\nu)=\varnothing$, and that $\mathcal{H}(\mu)$ and $\mathcal{H}(\nu)$ only contain one function. Then if $S=\nu+P(D) \mu$, it is shown in [1] that $S$ is invertible and in [4] that $\mathcal{H}(S)$ contains only one function, and therefore $S$ induces, by convolution, a surjection onto $D^{\prime}([0,+\infty))$. In particular, one could take $\nu$ to have a singular support constituted only by isolated points; see Corollary 16.3 .18 in [4].

REMARK. In case $\mathcal{H}(S)$ contains only one function, (1) implies that

$$
\operatorname{sing} \operatorname{supp}(S * u) \subseteq[j, a] \text { implies } a-j>\alpha,
$$

where the convex hull of $\operatorname{sing} \operatorname{supp}(S)$ is $[0, \alpha]$, and in $(1), b$ can be taken to be $a-\alpha$.

We now turn to the case of several variables. Let $\Gamma \subset \mathbf{R}^{n}$ be a closed convex cone whose vertex is the origin, and which does not contain any straight line. We denote by $D^{\prime}(\Gamma)$ the space of distributions supported in $\Gamma$, and by $D_{\Gamma}^{\prime}$ the space of distributions with support contained in some translate of $\Gamma$. It is well known [5] that if $S \in \mathcal{E}^{\prime}$ has support contained in $\Gamma$, then $S * D^{\prime}(\Gamma) \subseteq D^{\prime}(\Gamma)$. A complete characterization of those $S \in \mathcal{E}^{\prime}$ for which $S * D^{\prime}(\Gamma)=D^{\prime}(\Gamma)$ has been given by Hörmander [4], in Theorems 16.7.3, 16.7.4; in particular the condition asks that for every closed cone $\Gamma_{1} \subset \Gamma$, there are positive constants $C, M, A$ such that

$$
|1 / \hat{S}(z)|<C(1+|z|)^{M} \exp (A|\operatorname{Im} z|)
$$

if $\operatorname{Im} z \in \Gamma_{1}$ and $|\operatorname{Im} z|>C \log (2+|z|)$.

At first sight, it does not seem obvious to verify that every invertible distribution $S \in \mathcal{E}^{\prime}(\mathbf{R})$ for which $\mathscr{H}(S)$ consists only of one function satisfies (4), but this is indeed an immediate consequence of Theorem 1 and Hörmander's results.

The result of $[8]$, Theorem 1 , can be extended in an obvious way to the case of distributions supported in cones; the proof uses the same arguments as in [8], and the Lion-Titchmarsh theorems for supports of convolutions, [5]: for this reason it 
does not seem necessary to repeat it here; in this case the surjectivity condition is that $S$ must be invertible, and that

$$
\begin{aligned}
& \text { for all } a \in \mathbf{R}^{n} \text { and all } u \in D_{\Gamma}^{\prime}, \operatorname{sing} \operatorname{supp}(S * u) \subseteq \Gamma+\{a\} \text { implies } \\
& \text { sing } \operatorname{supp}(u) \subseteq \Gamma+\{a\} \text {. }
\end{aligned}
$$

However, it is easy to show that, in (5), one cannot replace $D_{\Gamma}^{\prime}$ with $\mathcal{E}^{\prime}$. To prove it, one simply considers any partial differential operator $P(D)$ which is not hyperbolic with respect to $\Gamma$. Then it is obvious that condition (5) with $D_{\Gamma}^{\prime}$ replaced by $\mathcal{E}^{\prime}$ is satisfied, while (5) itself is not.

2. In this section $\Gamma$ will be an open convex cone, with vertex at the origin, and such that it does not contain any straight line; denote by $\Gamma^{*}$ its dual cone $\Gamma^{*}=\{\xi \in$ $\mathbf{R}^{n}:\langle\xi, x\rangle \geq 0$ for all $\left.x \in \Gamma\right\}$, and let $S^{\prime}\left(\Gamma^{*}\right)$ be the space of tempered distributions with supports contained in the closed cone $\Gamma^{*}$. Consider $S_{1}, \ldots, S_{m} \in S^{\prime}\left(\Gamma^{*}\right)$ : we look for necessary and sufficient conditions on $S_{1}, \ldots, S_{m}$ in order to ensure the existence of $E_{1}, \ldots, E_{m} \in S^{\prime}\left(\Gamma^{*}\right)$ such that

$$
S_{1} * E_{1}+\cdots+S_{m} * E_{m}=\delta .
$$

It is well known [9] that the Laplace transform is an isomorphism between $S^{\prime}\left(\Gamma^{*}\right)$ and the space $H(\Gamma)$ of functions $f$ holomorphic in $\mathbf{R}^{n}+i \Gamma$ for which there are positive constants $\alpha, \beta, M$ such that

$$
|f(z)| \leq M\left(1+|z|^{2}\right)^{\alpha}\left(1+\Delta^{-\beta}(\operatorname{Im} z)\right), \quad \text { for all } z \in \mathbf{R}^{n}+i \Gamma,
$$

where $\Delta(y)=\operatorname{dist}(y, \partial \Gamma)$.

THEOREM 2. Given $S_{1}, \ldots, S_{m} \in S^{\prime}\left(\Gamma^{*}\right)$, a necessary and sufficient condition for the existence of $E_{1}, \ldots, E_{m}$ in $S^{\prime}\left(\Gamma^{*}\right)$ such that $(6)$ holds, is that there exists positive constants $C, \gamma, \delta$ such that, for all $z \in \mathbf{R}^{n}+i \Gamma$,

$$
\left|\hat{S}_{1}(z)\right|+\cdots+\left|\hat{S}_{m}(z)\right| \geq C\left(1+|z|^{2}\right)^{-\gamma}\left(1+\Delta^{-\delta}(\operatorname{Im} z)\right)^{-1} .
$$

PROOF. By taking the Laplace transform of (6), the necessity of (7) is obvious. We only sketch the proof of its sufficiency, since it follows the well-known lines of [3]. First notice that even if $H(\Gamma)$ is not an $A_{p}$ space in the sense of $[3]$, it does satisfy the properties which are necessary to apply the $\bar{\partial}$-techniques; indeed, since $\Gamma$ is convex, the function $\log \left(1+\Delta^{-\beta}(\operatorname{Im} z)\right)$ is plurisubharmonic; on the other hand, using the Laplace transform, one immediately sees that if $f \in H(\Gamma)$ its derivatives also belong to $H(\Gamma)$. In order to conclude, as in $[\mathbf{3}]$, with the construction of a suitable Koszul complex, it is sufficient to show that if an analytic function $f$ satisfies an $L^{2}$-estimate of the form

$$
\int_{\mathbf{R}^{n}+i \Gamma}|f(z)|^{2}\left(1+|z|^{2}\right)^{-\gamma}\left(1+\Delta^{-\delta}(\operatorname{Im} z)\right)^{-1} d \lambda<+\infty
$$

where $d \lambda$ is the Lebesgue measure, then $f \in H(\Gamma)$. To prove this, it is sufficient to apply the mean value theorem to $f$ on a ball centered at $z$ and of radius $\Delta(\operatorname{Im} z) / 2$, and then to use the Cauchy-Schwarz inequality.

\section{REFERENCES}

1. W. Abramczuk, A class of surjective convolution operators, Pacific J. Math. 110 (1984), 1-7.

2. L. Ehrenpreis, Solutions of some problems of division. IV, Amer. J. Math. 82 (1960), 522-588. 
3. L. Hörmander, Generators for some rings of analytic functions, Bull. Amer. Math. Soc. 73 (1967), 943-949.

4. _ The analysis of linear partial differential operators. II, Grundlehren Math. Wiss., SpringerVerlag, Berlin-Heidelberg-New York, 1983.

5. J. L. Lions, Supports dans la transformation de Laplace, J. Analyse Math. 2 (1952-53), 369-380.

6. L. Schwartz, Théorie des distributions. II, Hermann, Paris, 1959.

7. R. Shambayati and R. Zielezny, On Fourier transforms of distributions with one-sided bounded support, Proc. Amer. Math. Soc. 88 (1983), 237-243.

8. _ Convolution equations in spaces of distributins with one-sided bounded support, Trans. Amer. Math. Soc. 289 (1985), 707-713.

9. V. S. Vladimirov, Generalized functions in mathematical physics, Mir, Moscow, 1979.

10. M. R. Wohlers and E. J. Beltrami, Distributions and the boundary values of analytic functions, Academic Press, New York and London, 1966.

DÉPARTEMENT DE MATHÉMATIQUES, UNIVERSITÉ DE BORDEAUX I, 351 COURS DE LA LIBERATION, 33405 TALENCE, FRANCE

Scuola Normale Superiore, Piazza dei Cavalieri, 7, 56100, Pisa, Italy (Current address of D. C. Struppa)

Current address (Alex Meril): Section de Mathématiques, Université Antilles-Guyane, Fouillole, 97110 Point-à-Pitre, Guadeloupe, Antilles Françaises 